Ann. Biol. anim. Bioch. Biophys., I968, 8 (I), 69-79.

\title{
INFLUENCE DE LA QUALITÉ DE L'ENSILAGE SUR L'ÉVOLUTION DE LA CÉTONÉMIE POST-PRANDIALE CHEZ LE MOUTON
}

\author{
R. PUECH, J.-L. TISSERAND, S.-Z. ZELTER \\ avec la collaboration technique de F. De Monredon, Nicole Picot, Marguerite Naville. \\ Christiane Dumay, Catherine TaRdI \\ Laboratoire de Recherches sur la Conservation et l'Efficacité des Aliments, \\ 16, rue Claude-Bernard, Paris $5^{\mathrm{e}}$, \\ Centre national de Recherches zootechniques, \\ Institut national de la Recherche agronomique
}

\section{SOMMAIRE}

Nous avons étudié comparativement l'influence du foin et d'ensilages de bonne et mauvaise qualité sur la variation postprandiale de la cétonémie chez le mouton, dans deux expériences comportant chacune deux périodes avec inversion des régimes.

L'ingestion d'ensilage provoque une hypercétonémie d'autant plus marquée que l'aliment est de plus mauvaise qualitê. Cette hypercétonémie est faible avec le foin. Elle se résorbe quel que soit le régime en moins de 5 heures et en aucun cas ne s'accompagne d'hypoglycémie.

L'excrétion des corps cétoniques dans l'urine reflète les concentrations sanguines. Elle est plus élevée avec les mauvais ensilages.

L'hypercétonémie pourrait être due à une orientation des fermentations du rumen vers la production d'acide butyrique. Cet acide est cétogène et les quantités trouvées dans le rumen 2 heures après le début du repas sont plus élevées avec les ensilages qu'avec le foin.

Un dérèglement métabolique caractérisé par une élévation de la cétonémie (teneur du sang en corps cétoniques : acétone, acide acétoacétique et acide $\beta$-hydroxybutyrique) et un abaissement de la glycémie apparaît souvent chez les femelles des ruminants domestiques. Sous sa forme clinique c'est la toxémie de gestation de la brebis et l'acétonémie de la vache laitière. Parmi les causes supposées une carence énergétique qui occasionne une utilisation anormale ou incomplète des lipides de réserve mobilisés, créerait un terrain favorable à l'apparition de ces troubles nutritionnels. Mais une perturbation du métabolisme des produits terminaux de la digestion dans le rumen pourrait également les déclencher. Leur fréquence semblerait 
d'autre part augmenter plus spécialement chez les vaches laitières consommant systématiquement des ensilages. Ce dernier fait a attiré notre attention en raison des conséquences zootechniques sérieuses qu'entraîne la maladie et de l'importance croissante prise par l'ensilage dans l'alimentation des bovins et des ovins.

Certains auteurs mettent effectivement en parallèle les deux phénomènes. Dès I938, BRouwER et DijKstra pensent que l'ensilage " de type butyrique " doit favoriser 1'apparition de l'acétonémie. KNODT et al. (I942) observent une élévation de la cétonémie en fin d'hiver chez des vaches laitières tecevant de "l'ensilage évolué ". UDALL (I95I) cite parmi les causes possibles de l'acétonémie la distribution d'ensilage et ADLER (I956) incrimine la teneur des ensilages en corps cétoniques. POTTS et KESSI,ER (I957) trouvent une corrélation élevée entre ce dernier facteur et 1'acétonémie.

Néanmoins, des études plus précises ont été réalisées en infusant dans le rumen, à l'état pur, des acides gras volatils, de l'acide lactique ou des corps cétoniques, isolément ou en association. Elles montrent que l'action sur la cétonémie et la glycémie est faible ou nulle avec l'acide acétique. L'acide propionique est anticétogène et glycoformateur (JoHnson, r955 Rook et al., I963 Schultz et al., I959, STORRy, I965). L'acide butyrique est en partie métabolisé dans la paroi du rumen ou dans le foie en acide $\beta$-hydroxybutyrique; il provoque toujours une hypercétonémie et parfois une hypoglycémie (Schultz et al, I949, Schultz et al., I95I, PENnington, I952, Johnson, I955, Annison et al., 957, Rook et al., I963, RAMSEY et al., I965).

L'action de l'acide lactique est discutée. D'après HuETER et al. (I956) le lactate de sodium serait absorbé tel quel. Mais pour la quasi-totalité des auteurs cela paraît fort peu probable. Il est bien plus vraisemblable que l'acide lactique soit métabolisé dans le rumen en acide propionique Johns, (I95I), et dans certaines conditions de milieu en un mélange de propionique-butyrique STORry et al., (I965).

Pour les concentrations les plus couramment observées (200 mg p. Ioo ml) 1'absorption est presque nulle et les produits de dégradation sont les acides propionique, butyrique et en quantité moindre acétique ; le rapport propionique/butyrique est tel qu'il y a une légère augmentation de la cétonémie et de la glycémie WAL,Do et al., (Ig6o).

L'infusion dans le rumen de corps cétoniques ne modifierait ni la glycémie ni la cétonémie SchulTz, (I95I).

Toutes ces substances se forment certes en quantités variables au cours des fermentations dans le rumen et dans l'ensilage. Mais les résultats connus à ce jour constituent plutôt une présomption qu'une preuve expérimentale évidente de l'existence de relation directe entre la consommation d'ensilage et le trouble nutritionnel de l'acétonémie. Les substances de l'ensilage qui en seraient responsables ne sont pas non plus identifiées avec certitude.

Ce problème requiert l'attention car la part des ensilages dans l'alimentation des ruminants croît sans cesse. Pour le clarifier nous avons réalisé deux expériences avec des moutons dans le but :

I $^{\circ}$ de préciser 1'effet comparé du foin et de la qualité de l'ensilage sur l'évolution postprandiale de la cétonémie et de la cétonurie ;

$2^{\circ}$ de tenter d'en identifier les métabolites responsables;

$3^{\circ}$ de voir si l'ingestion d'ensilage crée dans le rumen des conditions propices à l'élévation de la cétonémie. 


\section{PRO'TOCOLE D'EXPÉRIENCE}

\section{Animaux et régimes alimentaires}

Expérience 1. - Deux groupes similaires de 2 moutons mâles castrés de race Limousine et d'un poids moyen de $45 \mathrm{~kg}$ sont accoutumés à un ensilage courant pendant une dizaine de jours.

Au cours des 15 jours suivants, l'un des groupes consomme à la place un ensilage de qualité A et le second un ensilage de qualité $B$. Ces deux aliments expérimentaux sont constitués d'un mélange de graminés à prédominance de Ray-grass. Ils sont permutés pour une deuxième période de 5 jours.

Chaque animal reçoit quotidiennement deux repas de $45^{\circ} \mathrm{g}$ de matière sèche.

Expérience II. - Six moutons mâles castrés de race Ile-de-France et d'un poids moyen de $63 \mathrm{~kg}$ sont répartis en trois groupes comparables de 2 sujets ( $\mathrm{x}$ animal est pourvu d'une canule permanente de rumen). L'un des groupes consomme en permanence du foin de luzerne $(F)$. Un deuxième reçoit un ensilage naturel de luzerne $(\mathrm{N})$ et le troisième un ensilage préfané de luzerne $(\mathrm{P})$. Les trois aliments proviennent de la même coupe.

L'expérience comporte une phase préliminaire d'accoutumance à ces aliments d'une dizaine de jours et deux périodes expérimentales de 15 jours chacune.

Les ensilages $\mathrm{N}$ et $\mathbf{P}$ sont permutés pour la seconde période expérimentale.

Deux repas journaliers de $55^{\circ} \mathrm{g}$ de matière sèche sont alloués à chaque sujet.

Les refus sont pesés journellement et la ration ajustée d'après la quantité de matière sèche consommée la veille. Les échantillons d'ensilages destinés à l'analyse sont conservés en congélateur $\grave{a}-18^{\circ} \mathrm{C}$. Les ensilages se différencient nettement par leurs qualités ; teneur en acides butyrique et lactique (tabl. I.).

Les jours de mesures biologiques, la durée du repas est strictement limitée à I heure, dans les deux expériences.

\section{Mesures biologiques}

Cétonémie et glycémie. - Leur évolution est suivie, les $7^{\mathrm{e}}$, ro $^{\mathrm{e}}$ et $\mathrm{I} 4^{\mathrm{e}}$ jours de chaque période expérimentale, sur des prélèvements de sang faits dans la veine jugulaire, en présence de fluorure de sodium $(2 \mathrm{mg} / \mathrm{ml})$. Dans la première expérience les prises sanguines ont lieu juste avant le repas du matin (temps o) et 60,90 , I20 et I 80 minutes après le début du repas; dans la seconde expérience elles sont effectuées aux temps $0,60,120,180$ et 300 minutes. Le sang est déféqué le jour même et le surnageant congelé à - $18^{\circ} \mathrm{C}$ jusqu’à l'analyse.

Cétonurie. - Elle est mesurée dans l'expérience I, les jours de prise de sang : l'urine des 24 heures est récoltée sous une couche de toluène afin d'éviter toute évolution. Dans l'expérience II, la récolte des urines a lieu soit les jours des prises de sang soit le lendemain afin de ne pas la faire coïncider avec les prélèvements de contenu de rumen. Cette collecte se fait en 4 fractions : de $\circ$ à 3 heures après le début du repas, de 3 à 6 , de 6 à 9 et de 9 à 24 heures. La faiblesse de certaines fractions $(5 \circ \mathrm{ml})$ et la présence de toluène rendant difficile la détermination de la densité, les volumes des émissions urinaires sont mesurés à l'éprouvette en évitant la formation de mousse.

Nature des fermentations dans le rumen. - Des prélèvements de contenu de panse ont lieu par pompage direct à travers la fistule à o, 2, 4, 5, 6 et 7 heures après le début du repas. Les échantillons sont filtrés sur 6 épaisseurs de gaze et congelés à - $18^{\circ} \mathrm{C}$. roo $\mathrm{ml}$ d'une solution de polyéthylèneglycol à ro p. $100(\mathrm{P} / \mathrm{V})$ sont introduits dans le rumen 2 heures après le début du repas pour évaluer le volume de son contenu.

Les substances suivantes sont dosées dans les divers prélèvements :

- acides gras volatils, dans les contenus de panse et dans les ensilages ;

- acide lactique dans les ensilages;-corps cétoniques (acétone, acide acéto-acétique et acide $\beta$-hy. droxybutyrique) dans les ensilages, le foin, les contenus de panse, le sang et l'urine ;-sucres réducteurs dans le sang;-polyéthylène glycol dans les contenus de panse prélevés aux temps $4,5,6$, et 7 heures après le début du repas.

\section{Méthodes d'analyses}

Les acides gras volatils sont déterminés par chromatcgraphie de partage gaz liquide selon la méthode de James et Martin modifiée ZELTER et LEROY, (1958), l'acide lactique suivant la méthode de LEPPER (I938) dans l'expérience I et par la méthode de BARNETT (I95I) dans l'expérience II. Les sucres réducteurs sont dosés par la méthode de Somogyi (I945) et NeLSON(I944). Le dosage des corps cétoniques a posé un certain nombre de problèmes. Sa méthodologie est exposée dans une note annexe PuEch, (1967). 


\section{RÉSULTATS}

La composition des fourrages est donnée dans le tableau I. Le critère de qualité adopté est la teneur en acide butyrique, un bon ensilage devant en être exempt. Les ensilages $A$ et $P$ sont meilleurs que les ensilages $B$ et $N$.

TABLEAU I

Caractéristiques des fourrages

\begin{tabular}{|c|c|c|c|c|c|c|c|c|}
\hline & \multirow[b]{2}{*}{ M.S. \% } & \multicolumn{2}{|c|}{ Acides } & \multicolumn{2}{|c|}{ g $\%$ frais } & \multirow[b]{2}{*}{$\begin{array}{c}\text { Corps } \\
\text { cétoniques } \\
\text { totaux } \\
\text { (mg) } \\
\% \% \text { Frais }\end{array}$} & \multirow[b]{2}{*}{$\begin{array}{l}\beta \text {-hydroxy- } \\
\text { butyrique } \\
\% \text { du total }\end{array}$} & \multirow[b]{2}{*}{$\begin{array}{l}\text { mg corps } \\
\text { cétoniques } \\
\text { ingérés } \\
\text { par repas }\end{array}$} \\
\hline & & Acétique & $\begin{array}{l}\text { Propio- } \\
\text { nique }\end{array}$ & $\begin{array}{l}\text { Buty- } \\
\text { rique }\end{array}$ & Lactique & & & \\
\hline Expérience $I$ & & & & & & & & \\
\hline Ensilage $A \ldots \ldots$ & 26,0 & 4,74 & 0,19 & 0,47 & 18,2 & 0,48 & 84,2 & 560 \\
\hline Ensilage $B \ldots \ldots$ & 31,3 & 6,15 & 1,79 & 10,9 & 2,6 & 0,22 & 90,7 & 180 \\
\hline Expérience II & & & & & & & & \\
\hline Ensilage naturel(N) & 21,7 & 6,23 & 0,23 & 3,71 & 10,43 & 1,61 & 88,8 & 3300 \\
\hline Ensilage préfané(P) & 46,7 & 1,66 & 0 & 0 & 9,02 & 2,18 & 89,0 & 2200 \\
\hline Foin $(\mathrm{F}) \ldots \ldots \ldots$ & 86,5 & - & - & & - & 0,78 & 72,9 & 510 \\
\hline
\end{tabular}

Glycémie. - La glycémie varie peu après le repas, tableau 2 ; elle a tendance à s'élever mais la variation, due à l'heure de prélèvement, n'est jamais significative au seuil de $5 \mathrm{p}$. roo. Il n'y a pas de différence entre animaux soumis à des régimes différents. Les valeurs observées sont toujours "normales" et correspondent à celles données par MCCANDLESS et DYe (I950).

\section{TABLEAU 2}

Variation de la glycémie

Moyenne de 12 mesures et erreur-type ( $\mathrm{mg}$ p. $100 \mathrm{ml}$ )

\begin{tabular}{|c|c|c|c|c|c|c|}
\hline Temps $\rightarrow$ & $0 \mathrm{mn}$ & $60 \mathrm{mn}$ & $90 \mathrm{mn}$ & $120 \mathrm{mn}$ & $180 \mathrm{mn}$ & $300 \mathrm{mn}$ \\
\hline Expérience I & & & & & & \\
\hline Ensilage $A \ldots \ldots$ & $45,0 \pm 1,14$ & $44,6 \pm 1,53$ & $46,2 \pm 1,84$ & $46,8 \pm 1,83$ & $46,8 \pm 1,31$ & \\
\hline Ensilage $B \ldots \ldots \ldots$ & $43,2 \pm 0,98$ & $45,3 \pm 1,58$ & $48,4 \pm 1,96$ & $46,7 \pm 1,39$ & $46,3 \pm 1,11$ & \\
\hline Expérience II & & & & & & \\
\hline Ensilage naturel $(\mathrm{N})$.. & $43,8 \pm 1,44$ & $46,5 \pm 1,39$ & - & $46,0 \pm 2,23$ & $45,8 \pm 2,32$ & $49,1 \pm 2,19$ \\
\hline Ensilage préfané $(\mathrm{P})$.. & $41,9 \pm 1,53$ & $40,2 \pm 1,08$ & - & $41,9 \mp 1,52$ & $42,6 \mp 1,28$ & $44,3 \pm 2,00$ \\
\hline Foin (F) $\ldots \ldots \ldots \ldots$ & $42,8 \pm 0,95$ & $43,0 \pm 1,76$ & - & $46,7 \pm 1,74$ & $46,3 \pm 1,37$ & $46,7 \pm 0,95$ \\
\hline
\end{tabular}




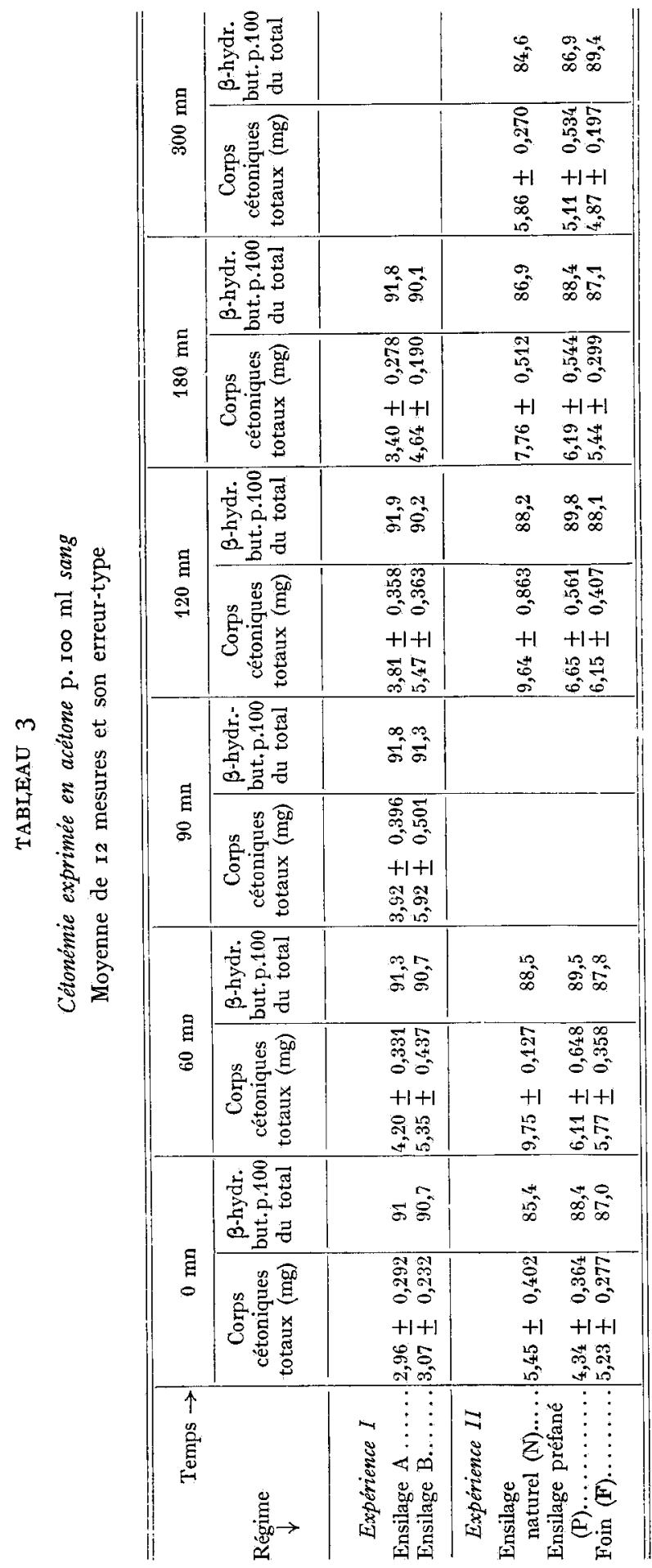


Cétonémie. - Elle augmente après le repas, passe par un maximum et revient au taux initial 5 heures après le début du repas. (tabl. 3).

\section{Influence de l'heure de prélèvement.}

La variation due à l'heure de prélèvement n'est pas significative pour le foin ; elle est significative $(P<0,05)$ pour les ensilages $B$ et $P$ et hautement significative pour les ensilages $A$ et $N(P<0,00 I)$.

\section{Influence du régime.}

Nous n'avons comparé les régimes qu'à l'intérieur de la même expérience car les niveaux de corps cétoniques au temps o sont différents d'une expérience à l'autre. Nous ne pouvons affirmer l'origine de cette différence, qui pourrait être en relation avec l'espèce fourragère : mélange de graminées dans l'expérience $I$, luzerne dans l'expérience II.

\section{Aux temps :}

- o mn, il n'y a pas de différence due au régime $(P>0,05)$, aussi bien dans la première que dans la deuxième expérience ;

- $60 \mathrm{mn}, 90 \mathrm{mn}$, I20 mn, I80 mn (exp. I), la différence entre les ensilages A (bon) et $\mathrm{B}$ (mauvais) est très significative $(\mathrm{P}<0, \mathrm{OI})$;

- $60^{\prime}, \mathrm{I} 2 \mathrm{O}^{\prime}, \mathrm{I} 8 \mathrm{o}^{\prime}$, (exp. II) il existe entre les 3 régimes une différence très significative $(\mathrm{P}<\mathrm{O}, \mathrm{OI})$ due à l'action plus marquée de l'ensilage naturel $(\mathrm{N})$ riche en acide butyrique donc mauvais. En effet, la différence entre l'ensilage préfané $(P)$, exempt d'acide butyrique, donc bon, et le foin est faible et jamais significative $(P>0,20)$.

- 300', les valeurs observées sont pratiquement identiques aux valeurs initiales et il n'y a plus de différence due au régime $(\mathrm{P}>0,05)$.

En somme, l'ingestion d'ensilage provoque une hypercétonémie postprandiale d'autant plus forte que l'ensilage est de plus mauvaise qualité, alors que le foin ne provoque pas d'hypercétonémie marquée. Il en résulte des différences de concentrations sanguines en corps cétoniques chez des animaux soumis à un régime de fourrages conservés suivant des techniques différentes.

Cette hypercétonémie postprandiale a déjà été observée sur vaches laitières recevant I $_{5} \mathrm{~kg}$ " d'ensilage évolué " par KNODT et al. (I942) : les teneurs citées pour l'ensilage et le foin sont respectivement de $3 \mathrm{mg}$ et $2,2 \mathrm{mg}$ p. Ioo $\mathrm{ml}$ avant le repas et de $5 \mathrm{mg}$ et $3 \mathrm{mg} \% \mathrm{ml}$ après le repas. Nos valeurs enregistrées sur les moutons sont plus élevées dans la deuxième expérience ; mais KNODT et al. ont expérimenté avec des régimes mixtes (fourrage + concentré).

D'ailleurs, nos animaux n'ont jamais présenté de symptômes apparents d'acétonémie et les valeurs avant le repas correspondent bien à celles d'animaux sains : I,5-2,2 mg p. IOO ml Sel,LERS et PUGH, (I95I) à 6,53 mg p. IOO ml KRONFELD, (I957).

Cétonurie (tab1. 4). - Les résultats sont exprimés en quantités totales de substances cétoniques (mg) excrétées par jour, car les grandes variations de volume urinaire observées $(0,4$ à $3,8 \mathrm{r} /$ jour $)$ nous amènent à penser que les concentrations ne reflètent pas fidèlement l'excrétion pondérale. Dans la première expérience, l'excrétion journalière moyenne est de $27 \mathrm{p}$. Ioo plus élevée avec l'ensilage $\mathrm{B}$ qu'avec 
l'ensilage $\mathrm{A}$. Cette différence est très significative. Si dans la deuxième expérience, nous attribuons la valeur Ioo au foin, les excrétions sont de I22 pour l'ensilage préfané (bon) et de 248 pour l'ensilage naturel (mauvais). Les excrétions urinaires avec les régimes de foin et d'ensilage préfané ne sont pas significativement différentes $(\mathrm{F}<\mathrm{I})$ alors qu'elles le sont hautement pour l'ensilage naturel $(\mathrm{P}<0,00 \mathrm{I})$.

\section{TABLEAU 4}

Corps cétoniques excrétés dans l'urine $\mathrm{mg} / \mathrm{jour}$

Moyenne 12 mesures et erreur-type

\begin{tabular}{|c|c|c|c|c|c|c|c|c|c|}
\hline \multicolumn{2}{|c|}{ Ensilage A } & \multicolumn{2}{|c|}{ Ensilage B } & \multicolumn{2}{|c|}{ Ensilage naturel } & \multicolumn{2}{|c|}{ Ensilage préfané } & \multicolumn{2}{|c|}{ Foin } \\
\hline $\begin{array}{l}\text { Corps } \\
\text { cétoniques } \\
\text { totaux } \\
\text { (mg) }\end{array}$ & $\begin{array}{c}\beta \text {-hydr. } \\
\text { but. } \% \\
\text { du } \\
\text { total }\end{array}$ & $\begin{array}{l}\text { Corps } \\
\text { cétoniques } \\
\text { totaux } \\
(\mathrm{mg})\end{array}$ & $\begin{array}{l}\text { B-hydr. } \\
\text { but. } \% \\
\text { du } \\
\text { total }\end{array}$ & $\begin{array}{c}\text { Corps } \\
\text { cétoniques } \\
\text { totaux } \\
(\mathrm{mg})\end{array}$ & $\begin{array}{c}\beta \text {-hydr. } \\
\text { but. } \% \\
\text { du } \\
\text { total }\end{array}$ & $\begin{array}{l}\text { Corps } \\
\text { cétoniques } \\
\text { totaux } \\
(\mathrm{mg})\end{array}$ & $\begin{array}{c}\beta \text {-hydr. } \\
\text { but. } \% \\
\text { du } \\
\text { total }\end{array}$ & $\begin{array}{l}\text { Corps } \\
\text { cétoniques } \\
\text { totaux } \\
(\mathrm{mg})\end{array}$ & $\begin{array}{c}\beta \text {-hydr. } \\
\text { but. } \% \\
\text { du } \\
\text { total }\end{array}$ \\
\hline $572 \pm 26$ & 83,9 & $726 \pm 63$ & 86,8 & $746 \pm 84$ & 90,4 & $36 \% \pm 32$ & 86,8 & $301 \pm 24$ & 84,0 \\
\hline
\end{tabular}

Corps cétoniques dans le rumen (tab1. 5). - Le volume du contenu du rumen déterminé $2 \mathrm{~h}$ après le début du repas à l'aide du polyéthylène-glycol est variable pour un même individu à un régime donné, si bien qu'il est difficile de mettre en évidence une différence due au régime.

La concentration du jus de rumen en corps cétoniques est sensiblement la mềme quelle que soit l'heure d'observation, et légèrement plus élevée avec l'ensilage préfané. Pour des quantités de corps cétoniques de 510, 2200 et $3300 \mathrm{mg}$ ingérées respectivement avec le foin, l'ensilage préfané et l'ensilage naturel, on retrouve dans le rumen, 2 heures après le début du repas les quantités suivantes calculées à partir du volume : 535 , 73I et $607 \mathrm{mg}$. Ceci implique une possibilité de disparition rapide.

TABLEAU 5

Corps cétoniques dans le jus de rumen $\mathrm{mg} \% \mathrm{ml}$

\begin{tabular}{|c|c|c|c|c|c|c|c|c|c|}
\hline \multirow[b]{2}{*}{ Régime } & \multicolumn{2}{|l|}{0} & \multicolumn{2}{|c|}{2 heures } & \multicolumn{2}{|c|}{5 heures } & \multicolumn{2}{|c|}{7 heures } & \multirow[b]{2}{*}{$\begin{array}{l}\text { Vol. moy. } \\
\text { rumen } 2 \mathrm{~h} \\
\text { (lit.) moy. } \\
6 \text { mesures }\end{array}$} \\
\hline & $\begin{array}{l}\text { Corps } \\
\text { cétoniques } \\
\text { totaux } \\
(\mathrm{mg})\end{array}$ & $\begin{array}{c}\beta \text {-hydr. } \\
\text { but. } \% \\
\text { du } \\
\text { total }\end{array}$ & $\begin{array}{l}\text { Corps } \\
\text { cétoniques } \\
\text { totaux } \\
(\mathrm{mg})\end{array}$ & $\begin{array}{c}\beta \text {-hydr. } \\
\text { but. } \% \\
\text { du } \\
\text { total }\end{array}$ & $\begin{array}{c}\text { Corps } \\
\text { cétcniques } \\
\text { totaux } \\
\text { (mg) }\end{array}$ & $\begin{array}{c}\beta \text {-hydr, } \\
\text { but. } \% \\
\text { du } \\
\text { total }\end{array}$ & $\begin{array}{l}\text { VCorps } \\
\text { cétoniques } \\
\text { totaux } \\
(\mathrm{mg})\end{array}$ & $\begin{array}{c}\beta \text {-hydr. } \\
\text { but. } \% \\
\text { du } \\
\text { total }\end{array}$ & \\
\hline $\begin{array}{l}\text { Ensilage } \\
\quad \text { naturel ... }\end{array}$ & 6,43 & 63,4 & 6,88 & 82,6 & 4,83 & 68,1 & 5,69 & 67,1 & $8,82 \pm 0,95$ \\
\hline $\begin{array}{l}\text { Ensilage pré- } \\
\text { fané } \ldots \ldots \\
\text { Foin ....... }\end{array}$ & 8,14 & 77,6 & 8,08 & 73,5 & 8,40 & 66,6 & 7,50 & 79,7 & $9,05 \pm 0,91$ \\
\hline Foin ........ & 4,73 & 68,7 & 6,19 & 68,7 & 5,87 & 63,4 & 5,98 & 67,6 & $8,65 \pm 0,67$ \\
\hline
\end{tabular}


Acides gras volatils dans le rumen. - La figure I montre que les concentrations en divers A. G. V. évoluent après le repas de la même façon quels que soient les régimes. Aucune différence n'est significative. Toutefois l'acide butyrique augmente plus après un repas d'ensilage. Il représente 2 heures après le début du repas, 7,4, II,6

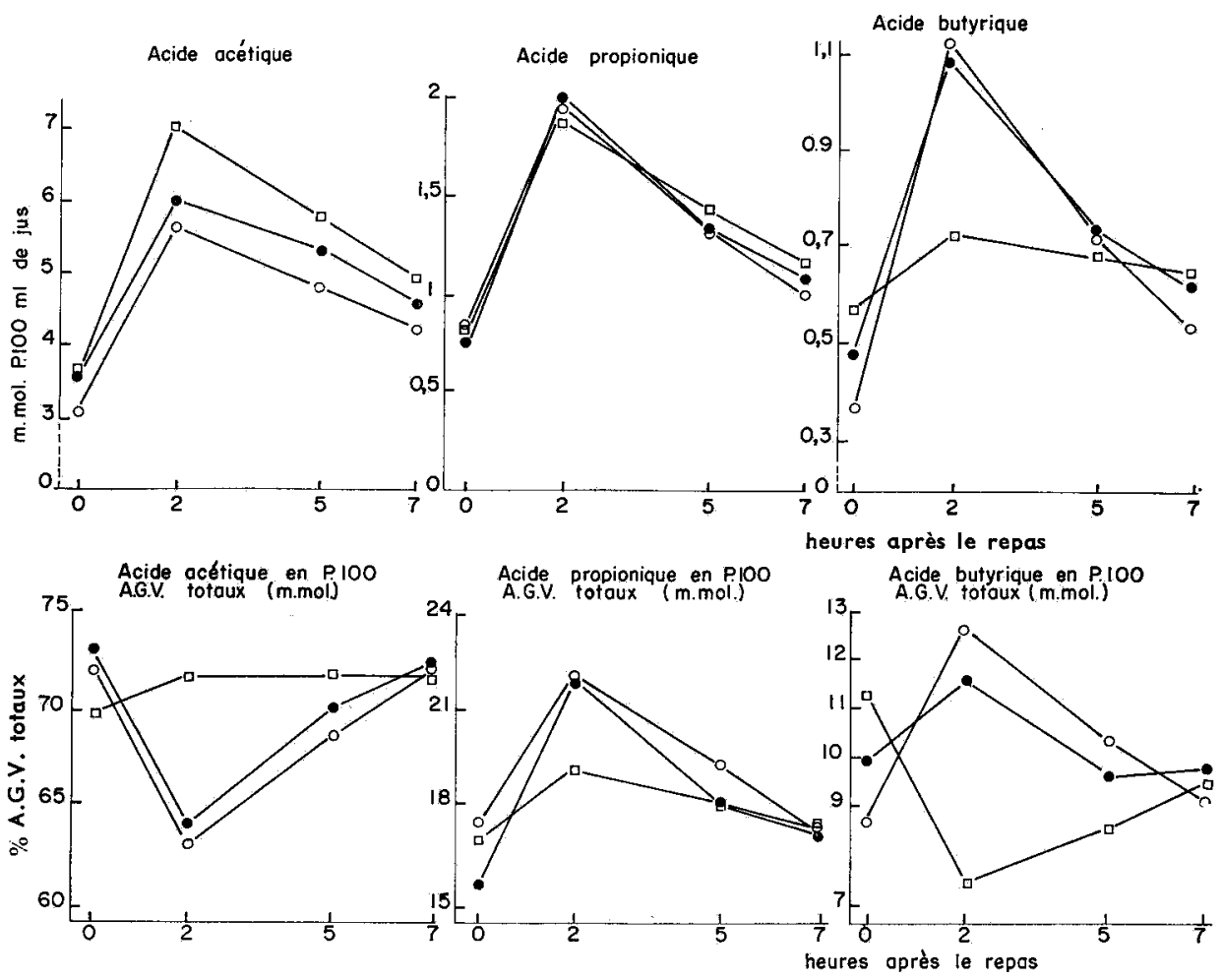

FIG. I.

$\square=$ Foin $\quad \bullet$ ens. préfané $\quad 0=$ ens. naturel.

et $\mathrm{I} 2,7 \mathrm{p}$. Ioo des A. G. V. totaux (en millimoles) respectivement pour le foin, l'ensilage préfané et l'ensilage naturel. La différence foin/ensilage approche la signification $(F=3,52$ alors que $F=3,68$ correspond à $P=0,05)$. Si au lieu des concentrations, on considére les quantités totales présentes dans le rumen 2 heures après le repas et on les rapporte à la matière sèche ingérée (tabl. 6), on constate que :

TABLEAU 6

A. G. V. présents dans le rumen $2 h$ après le repas (millimoles/100 g M. S. ingéré) 6 mesures

\begin{tabular}{|c|c|c|c|c|}
\hline & Acétique & Propionique & Butyrique & Valérianique \\
\hline $\begin{array}{l}\text { Ensilage naturel } \ldots \ldots \ldots \\
\text { Ensilage préfané......... } \\
\text { Foin } \ldots \ldots \ldots \ldots \ldots \ldots \ldots\end{array}$ & $\begin{array}{l}113 \pm 11,1 \\
110 \pm \quad 9,8 \\
111 \pm \quad 2,35\end{array}$ & $\begin{array}{l}40,5 \pm 4,3 \\
37,6 \pm 6,5 \\
30,2 \pm 0,75\end{array}$ & $\begin{array}{l}22,5 \pm 2,28 \\
19,8 \pm 2,26 \\
11,8 \pm 0,60\end{array}$ & $\begin{array}{l}3,8 \pm 0,95 \\
4,2 \pm 0,84 \\
2,8 \pm 0,58\end{array}$ \\
\hline
\end{tabular}


la variabilité des résultats est très faible pour le foin ; le P. E. G. semble donc être un marqueur de volume satisfaisant dans nos conditions expérimentales ; les quantités d'acides acétique et propionique sont sensiblement équivalentes avec tous les régimes, alors que la quantité $\mathrm{d}^{\prime}$ acide butyrique est très significativement plus faible $(\mathrm{P}<0$,or $)$ avec le foin qu'avec les ensilages.

\section{DISCUSSION}

Chez nos animaux d'expérience (mouton adulte à l'entretien), l'hypercétonémie postprandiale ne s'accompagne pas d'hypoglycémie, qui est l'une des caractéristiques sanguines de l'acétonémie. Il est toutefois possible qu'en présence d'un besoin élevé en glucose (pointe de lactation, fin de gestation) la glycémie s'abaisse et que, d'autre part, l'hypercétonémie postprandiale n'arrive pas à se réduire. C'est notamment le cas de l'expérience de KNODT et $a l$. (I954) sur vaches en lactation dont 1'hypercétonémie dure 9 heures, alors que dans la nôtre elle est réduite en 5 heures. L'on pourrait donc accuser les ensilages de favoriser l'apparition de l'acétonémie. Mais, dans notre expérience il n'y a jamais de différence significative entre foin et ensilage de bonne qualité. On ne saurait donc incriminer tous les ensilages, mais seulement ceux de mauvaise qualité.

Comment expliquer 1'hypercétonémie provoquée chez nos animaux par l'ingestion d'ensilage et quels en seraient les constituants responsables ? L'acide acétique n'est guère cétogène. L'acide propionique présent dans nos ensilages, est anticétogène. L'acide butyrique est certes cétogène, mais l'ensilage préfané n'en contient pas et provoque pourtant une augmentation de la cétonémie, bien moindre il est vrai, que les mauvais ensilages. Les corps cétoniques de l'ensilage ne peuvent expliquer cette augmentation car : dans la première expérience, l'ensilage le plus cétogène en renferme très peu (moins que le foin de la deuxième expérience). De plus, infusés à l'état pur dans le rumen, ils ne modifient pas la cétonémie Schulitz et Smirr, (I95I).

Serait-il plus logique de considérer non pas des substances apportées par les ensilages mais les métabolites qui en dérivent au cours des fermentations dans le rumen ? Parmi ces métabolites, il n'apparaît de différence nette que pour les concentrations d'acide butyrique. L'augmentation de la cétonémie avec les ensilages s'expliquerait déjà par la métabolisation de ce corps dans la paroi du rumen en acide B-hydroxybutyrique; d'autant plus que ce dernier représente dans nos expériences 80 à $90 \mathrm{p}$. Ioo des corps cétoniques sanguins totaux. L'évolution des concentrations postprandiales de même que les quantités totales de divers $\mathrm{A}$. G. V. présentes à un moment donné dans le rumen, accusent très nettement une différence pour l'acide butyrique, mais nous ne pouvons en affirmer l'origine. L'ensilage naturel apporte r6,7 millimoles d'acide butyrique "préformé " pour roo g de M. S. Ceci pourrait expliquer 22,5 millimoles d'acide butyrique trouvées dans le rumen pour roo $\mathrm{g}$ de M. S. ingérée au lieu de Ir,8 avec le foin. Mais l'ensilage préfané n'en apporte point ; il contient par contre une forte concentration en acide lactique dont le devenir dans le rumen est mal connu. On ne peut écarter a priori, l'idée que dans certaines conditions de milieu, il puisse servir de substrat pour la production d'acide butyrique par des microorganismes du rumen fermentant les lactates. Cette supposition s'ac- 
corde avec les observations in vitro de ZEITER (I953) et in vivo de WALDO et SCHULTZ (I960). Elle pourrait expliquer l'augmentation de la cétonémie enregistrée par nous après consommation d'un ensilage préfané totalement exempt d'acide butyrique, mais riche en acide lactique.

En somme, partant de nos conditions et données expérimentales et limitant leur interprétation au ruminant adulte à l'entretien, plus spécialement au mouton, il est possible de concevoir des processus distincts d'action des ensilages sur la cétonémie suivant leur qualité :

- les mauvais ensilages agiraient directement sur l'animal par leur fort apport d'acide butyrique exogène, substance connue depuis longtemps comme cétogène ;

- les bons ensilages exempts d'acide butyrique mais riches en acide lactique, exerceraient une action indirecte, en déviant l'activité métabolique de la microflore du rumen - lorsque les conditions de milieu s'y prêtent - vers une production dominante in situ d'acide butyrique, au liet d'acide propionique qui est la forme " normale " de métabolisation des lactates dans la panse.

Reçu pour publication en novembre 1967 .

\section{SUMMARY}

\section{INFLUENCE OF SILAGE QUALITY ON THE EVOLUTION}

OF POSTPRANDIAL KETONEMIA IN THE SHEEP

The comparative influence of hay and good or bad quality silages on the post prandial evolution of blood ketone bodies was estimated in two reversal experiments performed on sheep, some of them being rumen-fistulated.

Silage intake induced a ketonemia all the higher as the quality was lower (table 3 ); ketonemia remained at a lower level in hay-fed animals. It fell back to a normal level within 5 hours after the meal, whatever the diet; no simultaneous hypoglycemia was ever noticed.

The urinary ketone body excretion followed the same patterns as ketonemia. It was the highest with worst quality silages.

The hyperketonemia might be due to an increase in ruminal fermentations towards the production of butyric acid. For the amounts of this ketogenic acid found in the rumen two hours after the beginning of the meal were higher with silage than with hay.

\section{RÉFÉRENCES BIBLIOGRAPHIQUES}

AdLer J. H., 1956. Grass silage a possible nutritionel factor in bovine ketosis. Cornell Vet., 46, 446-45 . Annison E. F., Hill K. J., Lewis D., I 957. Studies on the portal blood of sheep 2. absorption of volatile fatty acids from the rumen of the sheep. Biochem. J., 68, 592-599.

Barnetr A. J. G., I95I. Determination colorimétrique de l'acide lactique dans l'ensilage. Biochem. J., 49, 527-534.

Brower E., Dijkstra N. D., 1938. An alimentary aretonemia and ketonemia in dairy cattle induced by feeding grass silage of the butyric acid type. J. Agric. Sci., 28, 695-702.

Hueter F. G., Shaw J. C., Doetsch R. N., 1956. Metabolism is the rumen. E. F. ANNISON and D. LEWIs. Methuen, London,

HyDEN S., I956. A turbidimetric method for the determination of higher poly ethylenegl ycols in materials Lantbrögsk Ann., 22, I 39-1 45 . 
Johnson R. B., I955. The effect of administration of acetic, propionic and N-butyric acids upon the blood glucose on ketone bodies levels of goats. Cornell Vet., 45, 273-279.

KNoDt C. B., Shaw J. C., White G. C., I942. Studies on ketosis in dairy cattle. Effect of stall and pasture feeding upon the concentration of blood and urinary acetone of dairy cattle. J. Dairy, Sci., 25, 837-850.

KRONFELD D. S.. I957. A comparison of normal concentrations of reducing sugars, volatile fatty acids and ketones bodies in the blood of lambs, pregnant ewes and non-pregnant adult ewes. Aust. J. Agric. Res., 8, 202-208.

LEPPER W. r938. , Techniques de dosage des acides acétique, butyrique et lactique dans les ensilages Tierernähr. u. futerimitl, 1, i 87-196.

Nelson W. L., I944. Photometric adaptation of the Somogyi methods for the determination of glucose. J. Biol. Chem., 153, 375-381.

Pennington R. J., 1952. The metabolism of Short-chain Fatty acids in sheep. Biochem. J., 51, $251-254$.

PotTs R. B., Kessier F. B. 1957. Effect of grass silage on milk flowers and blood and milk acetone bodies. J. Dairy $S c i ., 40,1466-1470$.

Puech R., 1968. Détermination des corps cétoniques dans des liquides biologiques. Ann. Biol. anim. Bioch. Biophys., 8, 81-85.

Ramsey H. A., Davis C. L., 1965. Metabolism of N-butyrate by the adult goat. J. Dairy, Sci., 48, 38I390.

Rook J. A., Batch C. C., Campling R. C., Fisher L. J., i963. The utilization of acetic, propionic and butyric acids by growing heifers. Brit. J. Nutr., 17, 399-406.

Schultz L. H., SMITH V. R., LARDY H. A., I948. The effect of the administration of various fatty acids on the blood ketone levels of ruminants. J. Anim. Sci., 7, 544-55I.

Schultz L. H., Smith V. R., I95I. Experimental alteration of the sugar and ketone levels of the blood of ruminants in relation to ketosis. J. Dairy $S c i ., 34$, I191-I I96.

Sellers K. C., Pugh P. D. S., 195I. The occurence of ketosis in pregnant ewes associated with parasitism. Vet. Rec., 63, 40-47.

Somogyi M., I945. A new reagent for the determination of sugars. J. Biol. Chem., 160, 6I-68.

Storry J. E., Rook J. A. F., I965. Effect in the cow of intramminal infusions of volatile fatty acids and of lactic acid on the secretion of the component fatty acids of the milk fat and on the composition of blood. Biochem., J., 06, 2 10-21 7 .

Udall D. H., 1951. Causes of acetonemia. North Amer. Vet., 32, 348-359.

Waldo D. R., Schultz L. H., I960. Blood and rumen changes following the intra-ruminal administration of glycogenic materials. J. Dairy Sci., 43, 496-505.

Zelter S. Z., Leroy F., r958. Azote uréique et activité bactérienne in vivo au niveau du rumen. Ann. Zootech., 7, $173-185$.

ZELTER S. Z., r953. Le rôle nutritionnel chez la vache en lactation, des acides acétique et butyrique formés au cours de l'ensilage. Ann. Zootech., 2, 303-364. 